PROCEEDINGS OF THE

AMERICAN MATHEMATICAL SOCIETY

Volume 125, Number 1, January 1997, Pages 235-241

S 0002-9939(97)03575-2

\title{
STABILITY AND ALMOST PERIODICITY OF SOLUTIONS OF ILL-POSED ABSTRACT CAUCHY PROBLEMS
}

\author{
R. DELAUBENFELS AND VŨ QUÔC PHÓNG \\ (Communicated by Palle E. T. Jorgensen)
}

\begin{abstract}
We give simple spectral sufficient conditions for a solution of the linear abstract Cauchy problem, on a Banach space, to be strongly stable or asymptotically almost periodic, without assuming that the associated operator generates a $C_{0}$-semigroup.
\end{abstract}

\section{INTRODUCTION}

Let $A$ be a closed linear operator on a Banach space $X$. We shall consider the many physical problems that may be modelled as an abstract Cauchy problem

$$
\left\{\begin{array}{l}
u^{\prime}(t)=A u(t), \quad t \geq 0, \\
u(0)=x
\end{array}\right.
$$

Many results on strong stability and almost periodicity of $t \mapsto u(t)$, when $A$ generates a $C_{0}$-semigroup, may be found in $[1,2,7,13,14,16,17]$ and [18], to name only a few references. The hypothesis that $A$ generate a $C_{0}$-semigroup is saying that (1) is well-posed.

We assume in this paper only that all exponentially bounded solutions of Eq. (1) are unique. We do not assume that $A$ generates a $C_{0}$-semigroup; this is what we mean by saying that (1) is ill-posed. Thus we are considering, as in [2], individual solutions of (1), but without any global well-posedness; our results are truly local in character.

When the spectrum of $A$ on the imaginary axis is countable, we characterize bounded uniformly continuous solutions of (1) that are asymptotically almost periodic, or strongly stable, in terms of the means of $t \mapsto e^{-\lambda t} u(t)$, for purely imaginary $\lambda$ in the spectrum of $A$ (Theorem 4). Theorem 5 is a local version of the theorem of Katznelson-Tzafriri for $C_{0}$-semigroups. Finally, when the spectrum of $A$ does not intersect the imaginary axis, we show that any uniformly continuous mild solution of (1) is strongly stable (Proposition 7).

Our technique is to use the Hille-Yosida space (see Section 2), introduced, independently, in [10] and [12], as constructed in [3, Chapter V], to deduce a local result from a global result. More specifically, if $t \mapsto u(t)$ is a bounded, uniformly continuous mild solution of (1), then $x$ is in the Hille-Yosida space, on which $A$

Received by the editors April 21, 1995 and, in revised form, August 4, 1995.

1991 Mathematics Subject Classification. Primary 47D06.

(C) 1997 American Mathematical Society 
generates a $C_{0}$-semigroup of contractions. We may then apply results about the asymptotic behaviour of orbits of a $C_{0}$-semigroup.

Throughout this paper, $A$ is a closed linear operator in a Banach space $X, T(t)$ is a $C_{0}$-semigroup in $X$. The spectrum, point spectrum and resolvent set of $A$ are denoted by $\sigma(A), P \sigma(A)$ and $\rho(A)$, respectively.

\section{Preliminaries}

We consider the abstract Cauchy problem (1), where $A$ is a closed linear operator on a Banach space $X$. We assume throughout the paper that $A$ is such that all exponentially bounded solutions (i.e. all solutions $u(t)$ such that $\|u(t)\| \leq M e^{\alpha t}$ for some $M$ and $\alpha$ ) of (1) are unique. For instance, it is enough to require that there exists $\lambda_{0} \in \mathbf{R}$ such that

$$
(\lambda-A) \text { is injective for } \lambda \geq \lambda_{0} \quad \text { (see [12]). }
$$

A function $u(t), t \geq 0$, is called a strong solution of Eq. (1), if $u \in C^{1}(\mathbf{R}, X), u(t)$ $\in \mathcal{D}(A)$ for all $t \geq 0$ and satisfies (1). To introduce the notion of Hille-Yosida space, we need the following definition of mild solutions of Eq. (1) (see [3]).

Definition. A continuous function $u: \mathbf{R}^{+} \rightarrow X$ is called a mild solution of Eq. $(1)$, if $v(t) \equiv \int_{0}^{t} u(s) d s \in \mathcal{D}(A)$ and satisfies $\frac{d}{d t} v(t)=A v(t)+x$, for all $t \geq 0$.

In the sequel, it will sometime be convenient to write this solution as $u(t, x)$.

Let $Z_{0}$ be the set of all $x$ for which Eq. (1) has a bounded uniformly continuous mild solution $u(t, x), t \geq 0$.

We will write $X \hookrightarrow Y$ to mean that there is a continuous embedding of $X$ into $Y$. If $A$ is an operator on $Y$, we will write $\left.A\right|_{X}$ to mean the restriction of $A$ to $X$. Finally, we introduce a norm in $Z_{0}$ by

$$
\|x\|_{Z_{0}} \equiv \sup _{t \geq 0}\|u(t, x)\| .
$$

Lemma 1. The space $Z_{0}$ has the following properties:

(1) $Z_{0}$ is a Banach space;

(2) $Z_{0} \hookrightarrow X$;

(3) $\left.A\right|_{Z_{0}}$ generates a $C_{0}$-semigroup of contractions $T(t)$, given by

$$
T(t) x \equiv u(t, x) \quad(t \geq 0)
$$

(4) $Z_{0}$ is maximal-unique, i.e., if $W$ is a Banach space such that $W \hookrightarrow X$ and $\left.A\right|_{W}$ generates a bounded $C_{0}$-semigroup, then $W \hookrightarrow Z_{0}$; and

(5) if $B \in B(X)$ and $B A \subseteq A B$, then $B \in B\left(Z_{0}\right)$, with $\|B\|_{Z_{0}} \leq\|B\|$.

Proof. The proof of (1)-(4) can be found in [10], or [3], so we need only to show (5). Since $B$ commutes with $A$, for any $x \in Z_{0}$,

$$
v(t) \equiv B u(t, x)(t \geq 0)
$$

is a mild solution of (1) with the initial value $v(0)=B x$; that is, $B Z_{0} \subseteq Z_{0}$, with

$$
\|B x\|_{Z_{0}} \equiv \sup _{t \geq 0}\|u(t, B x)\|=\sup _{t \geq 0}\|B u(t, x)\| \leq\|B\|\|x\|_{Z_{0}}\left(x \in Z_{0}\right) .
$$

The space $Z_{0}$, which was introduced in [12] and [10] (independently) is called the Hille-Yosida space for $A$ (cf. [3, 4]). 
From Lemma 1-(5) the following corollary immediately follows.

Corollary 2. $\sigma\left(\left.A\right|_{Z_{0}}\right) \subseteq \sigma(A)$.

\section{MAIN RESUlts}

Let $T(t)$ be a bounded $C_{0}$-semigroup, with generator $A$. It is known that the spectrum $\sigma(A)$ of $A$ is contained in the closed left half-plane $\{\lambda \in \mathbf{C}: \operatorname{Re} \lambda \leq 0\}$. For $\lambda \in i \mathbf{R}$, let $X_{\lambda}(A)=\{x \in X: A x=\lambda x\}$, and $X_{\lambda}\left(A^{*}\right)=\left\{\phi \in X^{*}: A^{*} \phi=\lambda \phi\right\}$. If $x \in X_{\lambda}(A)$ and $x \neq 0$, then, by a simple argument using the Markov-Kakutani Fixed Point Theorem, there exists $\phi \in X_{\lambda}\left(A^{*}\right)$ such that $\langle x, \phi\rangle \neq 0$. The semigroup $T(t)$ is called mean ergodic, if $\frac{1}{R} \int_{0}^{R} T(t) d t$ converges strongly as $R \rightarrow \infty$ (in this case the limit operator $P$ is the projection operator from $X$ onto the subspace $\{x \in X: T(t) x=x, \forall t \geq 0\}$ ). By the well known Mean Ergodic Theorem (see e.g. [8]), the semigroup $\{T(t)\}$ is mean ergodic if and only if $\operatorname{ker}(A)+\operatorname{ran}(A)$ is dense in $X$. It is easy to see that the latter condition is equivalent to the following: $X_{0}\left(A^{*}\right)$ separates points of $X_{0}(A)$, i.e. for every $\phi \in X_{0}\left(A^{*}\right)$ there exists $x \in X_{0}(A)$ such that $\langle x, \phi\rangle \neq 0$.

The semigroup $T(t)$ is called almost periodic, if the trajectory $\{T(t) x: t \geq 0\}$ is relatively compact in $X$ for every $x \in X$. It is well known that $T(t)$ is an almost periodic semigroup if and only if, for every $x \in X$, the function $u(t)=T(t) x, t \geq 0$, is asymptotically almost periodic, i.e. there exists an almost periodic function $v: \mathbf{R} \rightarrow X$ such that $\|u(t)-v(t)\| \rightarrow 0$ as $t \rightarrow \infty$. Moreover, if $T(t)$ is an almost periodic semigroup, then $e^{-\lambda t} T(t)$ is ergodic for every $\lambda \in i \mathbf{R}$ (consequently, $X_{\lambda}\left(A^{*}\right)$ separates points of $X_{\lambda}(A)$, for every $\left.\lambda \in P \sigma\left(A^{*}\right) \cap i \mathbf{R}\right)$. Let $P_{\lambda}$ denote the corresponding projection operator which is the strong limit, as $R \rightarrow \infty$, of $\frac{1}{R} \int_{0}^{R} e^{-\lambda t} T(t) d t$. Then $T(t)$ is strongly stable, i.e. $\|T(t) x\| \rightarrow 0$ as $t \rightarrow \infty$, $\forall x \in X$, if and only if it is almost periodic and $P_{\lambda}=0$ for every $\lambda \in P \sigma(A) \cap i \mathbf{R}$ (see $[5,16])$.

In [16] the following almost periodicity theorem has been established: if $\{T(t)\}$ is a bounded $C_{0}$-semigroup, such that $\sigma(A) \cap i \mathbf{R}$ is countable, and if $X_{\lambda}\left(A^{*}\right)$ separates points of $X_{\lambda}(A)$ for each $\lambda \in P \sigma\left(A^{*}\right) \cap i \mathbf{R}$, then $T(t)$ is almost periodic.

From this theorem we obtain the following result on almost periodicity of individual trajectories.

Proposition 3. Let $\{T(t)\}_{t \geq 0}$ be a bounded $C_{0}$ semigroup with generator $A$ such that $\sigma(A) \cap i \mathbf{R}$ is countable, and let $x$ be a vector in $X$. Then

(i) the function $u(t)=T(t) x$ is asymptotically almost periodic if (and only if) for each $\lambda \in \sigma(A) \cap i \mathbf{R}$ the function $e^{-\lambda t} u(t)$ has convergent means; and

(ii) the function $u(t)$ converges to zero strongly as $t \rightarrow \infty$ if (and only if) for each $\lambda \in \sigma(A) \cap i \mathbf{R}$, the function $e^{-\lambda t} u(t)$ has convergent means with the limit equal to 0 .

Proof. (i) Let $Y=\overline{\operatorname{span}}\{T(t) x: t \geq 0\}$. Then $Y$ is a closed invariant subspace of $T(t)$. It is easy to see that $\sigma\left(\left.A\right|_{Y}\right) \cap i \mathbf{R} \subseteq \sigma(A) \cap i \mathbf{R}$, so it is countable. From the condition it follows that

$$
\frac{1}{R} \int_{0}^{R} e^{-\lambda} T(t) y d t
$$

converges strongly, as $R \rightarrow \infty$, for each $y \in \operatorname{span}\{T(t) x: t \geq 0\}$, and hence it also converges strongly for each $y \in \overline{\operatorname{span}}\{T(t) x: t \geq 0\}$. Therefore, by the quoted 
almost periodicity theorem of Lyubich and Vũ Quôc Phóng [16], $\left.T(t)\right|_{Y}$ is an almost periodic semigroup, hence $T(t) x$ is an asymptotically almost periodic function.

(ii) If, in addition,

$$
\lim _{R \rightarrow \infty} \frac{1}{R} \int_{0}^{R} e^{-\lambda t} u(t) d t=0
$$

for all $\lambda \in P \sigma(A) \cap i \mathbf{R}$, then

$$
\lim _{R \rightarrow \infty} \frac{1}{R} \int_{0}^{R} e^{-\lambda t} T(t) y d t=0
$$

for all $y \in Y$, which implies that $P_{\lambda}\left(\left.A\right|_{Y}\right)=0$ for all $\lambda \in P \sigma\left(\left.A\right|_{Y}\right) \cap i \mathbf{R}$, so that $T(t) y \rightarrow 0$ for all $y \in Y$. In particular, $u(t) \rightarrow 0$ as $t \rightarrow \infty$.

Using Proposition 3 and the Hille-Yosida space, we obtain the following result, where we say that a function $\omega$ has uniformly convergent means if

$$
\lim _{T \rightarrow \infty} \frac{1}{R} \int_{a}^{R+a} \omega(s) d s
$$

exists, uniformly in $a \geq 0$.

Theorem 4. Suppose $\sigma(A) \cap i \mathbf{R}$ is countable and $u(t), t \geq 0$, is a bounded uniformly continuous mild solution of Eq. (1). Then

(1) $u(t)$ is asymptotically almost periodic if (and only if) for every $\lambda \in \sigma(A) \cap i \mathbf{R}$, the function $e^{-\lambda t} u(t)$ has uniformly convergent means;

(2) $u(t)$ converges strongly to 0 as $t \rightarrow \infty$ if (and only if) for each $\lambda \in \sigma(A) \cap i \mathbf{R}$, the function $e^{-\lambda t} u(t)$ has uniformly convergent means with the limit equal to 0 .

Proof. Let $Z_{0}$ be the Hille-Yosida space and $T(t)$ be the semigroup generated by $\left.A\right|_{Z_{0}}$. By Corollary 2, $\sigma\left(\left.A\right|_{Z_{0}}\right) \cap i \mathbf{R}$ is countable. We will show that, for $\lambda \in$ $\sigma(A) \cap i \mathbf{R}, \quad e^{-\lambda t} T(t) x=e^{-\lambda t} u(t)$ has uniformly convergent means as a function from $\mathbf{R}^{+}$to $Z_{0}$.

Fix $\epsilon>0$. There exists $T_{\epsilon}$ such that

$$
\left\|\frac{1}{T} \int_{h}^{T+h} e^{-\lambda t} u(t) d t-\frac{1}{S} \int_{h}^{S+h} e^{-\lambda t} u(t) d t\right\|<\epsilon,
$$

for all $S, T>T_{\epsilon}, h>0$. From (2) it follows that

$$
\begin{aligned}
& \left\|\frac{1}{T} \int_{h}^{T+h} e^{-\lambda t} u(t) d t-\frac{1}{S} \int_{h}^{S+h} e^{-\lambda t} u(t) d t\right\|_{Z_{0}} \\
& \equiv \sup _{s \geq 0}\left\|u\left(s, \frac{1}{T} \int_{h}^{T+h} e^{-\lambda t} u(t) d t-\frac{1}{S} \int_{h}^{S+h} e^{-\lambda t} u(t) d t\right)\right\| \\
& =\sup _{s \geq 0}\left\|\frac{1}{T} \int_{h}^{T+h} e^{-\lambda t} u(t+s) d t-\frac{1}{S} \int_{h}^{S+h} e^{-\lambda t} u(t+s) d t\right\| \\
& =\sup _{s \geq 0}\left\|\frac{1}{T} \int_{h+s}^{T+h+s} e^{-\lambda t} u(t) d t-\frac{1}{S} \int_{h+s}^{S+h+s} e^{-\lambda t} u(t) d t\right\| \\
& \leq \epsilon
\end{aligned}
$$

since the convergence is uniform in $h$. 
Thus $e^{-\lambda t} u(t)=e^{-\lambda t} T(t) x$ has uniformly convergent means, for any $\lambda \in$ $\sigma\left(\left.A\right|_{Z_{0}}\right) \cap i \mathbf{R}$. Now the statements (1)-(2) follow from Proposition 3 and the continuous embedding $Z_{0} \hookrightarrow X$.

Theorem 4 is a generalization of the above mentioned result of Lyubich and V $\tilde{u}$ Quôc Phóng [16], but the proof is based on this result and the Hille-Yosida space.

Part (2) of Theorem 4 is analogous to, but independent of, [2, Theorem 1].

An important corollary of this result, which was obtained independently by Arendt and Batty [1] (see also [14]) (and is sometimes known as the ABLP Theorem), states that $\|T(t) x\| \rightarrow 0$ as $t \rightarrow \infty$ for all $x$ in $X$, if $\sigma(A) \cap i \mathbf{R}$ is countable and $\operatorname{P\sigma }\left(A^{*}\right) \cap i \mathbf{R}$ is empty.

We note that the results presented in Theorem 4 are new even for the case when $A$ is a generator of a $C_{0}$-semigroup (and even for bounded $A$ ). In this case, Theorem 4-(1) (resp., (2)) gives a condition for asymptotic almost periodicity (resp., stability) of individual trajectories of $C_{0}$-semigroups.

Our next result is an individual version of the theorem of Katznelson-Tzafriri type obtained in $[7,17]$ (independently) for $C_{0}$-semigroups. A function $f \in L^{1}(\mathbf{R}$ ) is said to be a function of spectral synthesis with respect to a closed subset $\triangle$ of $\mathbf{R}$ if there is a sequence $g_{n} \in L^{1}(\mathbf{R})$, such that, for each $n, \hat{g}_{n}$ vanishes in a neighborhood of $\triangle$ and $\left\|g_{n}-f\right\|_{L^{1}} \rightarrow 0$ as $n \rightarrow \infty$.

Theorem 5. Suppose that $u(t), t \geq 0$, is a bounded uniformly continuous mild solution of Eq. (1) and $f \in L^{1}\left(\mathbf{R}_{+}\right)$is a function of spectral synthesis with respect to $-i \sigma(A) \cap \mathbf{R}$. Then

$$
\lim _{t \rightarrow \infty}\left\|\int_{0}^{\infty} f(s) u(t+s) d s\right\|=0 .
$$

Proof. Again consider the Hille-Yosida space $Z_{0}$ and the semigroup $T(t)$ generated by $\left.A\right|_{Z_{0}}$. By $[7,17]$,

$$
\lim _{t \rightarrow \infty}\left\|\int_{0}^{\infty} f(s) T(t+s) x d s\right\|_{Z_{0}}=0,
$$

from which (3) immediately follows, since $Z_{0} \hookrightarrow X$.

Theorem 5 is a generalization of a result obtained independently by Vũ Quôc Phóng [17] and Esterle, Strouse and Zouakia [7], which states that if $T(t)$ is a bounded $C_{0}$-semigroup with generator $A$ and if $f \in L^{1}\left(\mathbf{R}_{+}\right)$is a function of spectral synthesis with respect to $(-i \sigma(A) \cap \mathbf{R})$, then

$$
\lim _{t \rightarrow \infty}\left\|\int_{0}^{\infty} f(s) T(t+s) d s\right\|=0 .
$$

This result is an extension of an analogous result obtained by Katznelson and Tzafriri [11] for power-bounded operators.

From Theorem 5 we have the following corollary (here $\hat{u}$ denotes the Laplace transform of $u$, i.e. $\left.\hat{u}(\lambda)=\int_{0}^{\infty} e^{-\lambda t} u(t) d t, \operatorname{Re} \lambda>0\right)$.

Corollary 6. If $\sigma(A) \cap i \mathbf{R} \subseteq\{0\}$ and $u(t)$ is a bounded uniformly continuous mild solution of Eq. (1), then

$$
\lim _{t \rightarrow \infty}\left\|\hat{u}_{t+s}(\lambda)-\hat{u}_{t}(\lambda)\right\|=0, \forall s \geq 0, \operatorname{Re} \lambda>0 .
$$


In conclusion, we give the following proposition, which gives another simple condition for stability of individual solutions. Assertion (3) is a special case of [9, Theorem 2.5]. Assertion (1) may be deduced from [2, Theorem 1] by the techniques of this paper, by going down to the Hille-Yosida space for $A-\omega$, for any $\omega>0$. However, the referee has pointed out that an inspection of the proof of $[2$, Theorem $1]$ shows that the proof applies, without change, when $A$ does not generate a strongly continuous semigroup.

Proposition 7. Suppose $\sigma(A) \cap i \mathbf{R}$ is empty.

(1) If $u$ is a uniformly continuous mild solution of Eq. (1), then $\lim _{t \rightarrow \infty} u(t)=0$.

(2) If $u$ is a bounded mild solution on $\mathbf{R}^{+}$of Eq. (1), then $\lim _{t \rightarrow \infty} A^{-1} u(t)=0$.

(3) There does not exist a nontrivial bounded mild solution on $\mathbf{R}$ of Eq. (1).

Proof. (1) As we commented above, the proof of [2, Theorem 1] is valid under the hypotheses of this theorem.

(2) From the conditions it follows that $A^{-1} u(t)$ is a bounded uniformly continuous mild solution of Eq. (2), thus (2) follows from assertion (1).

(3) Suppose $u \neq 0$ is a bounded mild solution on $\mathbf{R}$ of Eq. (1). Then $A^{-1} u(t)$ is a nontrivial bounded uniformly continuous mild solution on $\mathbf{R}$ of this equation. By [18, Proposition 3.7], and Corollary 2, $\operatorname{Sp}\left(A^{-1}(u)\right) \subset \sigma\left(\left.A\right|_{Z}\right) \cap i \mathbf{R} \subseteq \sigma(A) \cap i \mathbf{R}$, which is a contradiction to $\sigma(A) \cap i \mathbf{R}=\emptyset$.

Results similar to Proposition 7(1), when $A$ generates a bounded once integrated semigroup, may be found in [6, Theorem 5.6 and Corollary 5.9]. When $\sigma(A) \cap i \mathbf{R}$ is empty, [6, Theorem 5.6 and Corollary 5.9] follow immediately from Proposition $7(1)$, since, as mentioned in [6], $u(t) \equiv S(t) A x+x$ is a solution of (1) when $S(t)$ is a once integrated semigroup generated by $A$. However the results in $[6$, Theorem 5.6 and Corollary 5.9] have weaker hypotheses, analogous to [1] and [14]: $\sigma(A) \cap i \mathbf{R}$ is countable, $P \sigma\left(A^{*}\right) \cap i \mathbf{R}$ is empty and $0 \in \rho(A)$.

We should also remark that stability results for regularized semigroups (see [3]) also follow immediately from Proposition 7(1): if $\sigma(A) \cap i \mathbf{R}$ is empty, and $A$ generates a bounded regularized semigroup $\{W(t)\}_{t \geq 0}$, then $W(t) x \rightarrow 0$, as $t \rightarrow \infty$, for all $x \in X$.

\section{REFERENCES}

1. W. Arendt and C.J.K. Batty, Tauberian theorems and stability of one-parameter semigroups, Trans. Amer. Math. Soc. 306 (1988), 837-852. MR 89g:47053

2. C.J.K. Batty and Vũ Quôc Phóng, Stability of individual elements under one-parameter semigroups, Trans. Amer. Math. Soc. 322 (1990), 805-818. MR 91c: 47072

3. R. deLaubenfels, Existence families, functional calculi and evolution equations. Lecture Notes in Math., vol. 1570, Springer, Berlin, 1994. MR 96b:47047

4. R. deLaubenfels and S. Kantorovitz, Laplace and Laplace-Stieltjes spaces, J. Functional. Anal. 116 (1993), 1-61. MR 94g:47015

5. K. DeLeeuw and I. Glicksberg, Applications of almost periodic compactifications, Acta Mathematica 105 (1961), 63-97. MR 24:A1632

6. O. ElMennaoui, Asymptotic behaviour of integrated semigroups, J. Comp. Appl. Math. 54 (1994), 351-369. MR 96a:47067

7. J. Esterle, E. Strouse and F. Zouakia, Stabilité asymptotique de certains semigroupes dópérateurs, J. Operator Theory 28 (1992), 203-227. MR 95f:43001

8. J.A. Goldstein, Semigroups of linear operators and applications, Oxford Univ. Press, Oxford, 1985. MR 87c:47056

9. S. Huang, Characterizing spectra of closed operators through existence of slowly growing solutions of their Cauchy problems, Studia Math. 116 (1995), 23-41. CMP 96:02 
10. S. Kantorovitz, The Hille-Yosida space of an arbitrary operator, J. Math. Anal. and Appl. 136 (1988), 107-111. MR 90a:47097

11. Y. Katznelson and L. Tzafriri, On power-bounded operators, J. Functional Analysis 68 (1986), 313-328. MR 88e:47006

12. S.G. Krein, G.I. Laptev and G.A. Cvetkova, On Hadamard correctness of the Cauchy problem for the equation of evolution, Soviet Math. Dokl. 11 (1970), 763-766. MR 42:637

13. B.M. Levitan and V.V. Zhikov, Almost periodic functions and differential equations, Cambridge Univ. Press, Cambridge, 1982. MR 84g:34004

14. Yu.I. Lyubich and Vũ Quôc Phóng, Asymptotic stability of linear differential equations on Banach spaces, Studia Math. 88 (1988), 37-42. MR 89e:47062

15. R. Nagel, One-parameter semigroups of positive operators. Lecture Notes in Math. 1184, Springer, Berlin, 1986. MR 88i:47022

16. Vũ Quôc Phóng and Yu.I. Lyubich, A spectral criterion for almost periodicity of oneparameter semigroups, J. Soviet Math. 48 (1990), 644-647. Originally published in: "Teor. Funktsii, Funktsional. Anal. i Prilozhenia", 47, 36-41 (1987). MR 89a:47067

17. Vũ Quôc Phóng, Theorems of Katznelson-Tzafriri type for semigroups of operators, J. Functional Analysis 103 (1992), 74-84. MR 93e:47050

18. Vũ Quôc Phóng, On the spectrum, complete trajectories and asymptotic stability of linear semidynamical systems, J. Differential Equations 105 (1993), 30-45. MR 94f:47049

Department of Mathematics, Ohio University, Athens, Ohio 45701

E-mail address, R. deLaubenfels: 72260.2403@compuserve.com

E-mail address, Vũ Quôc Phóng: qvu@oucsace.cs.ohiou.edu 